\title{
Smart resource allocation needed to study 'legal highs'
}

Jeffery H Moran

\begin{abstract}
Ground-breaking science in the area of cannabinoid signaling holds great promise for new drug development, but an unintended consequence of this research has led to the emergence of an onslaught of deadly 'legal highs'. Providing public health scientists with access to laboratory resources designed to investigate emerging chemicals of concern is crucial for timely, sound evaluation of products that pose major threats to public health.
\end{abstract}

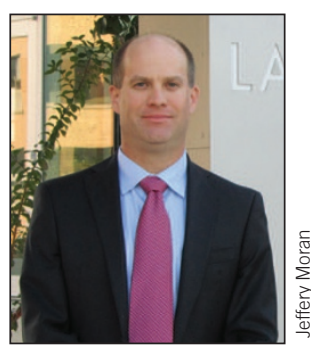

Marijuana has been used medicinally and recreationally for centuries, but scientists did not identify its principal psychoactive component tetrahydrocannabinol (THC) until 1964. Since this discovery, researchers have investigated many other natural and synthetic cannabinoids as potential therapeutic agents. Increasingly, these experimental compounds are ending up in the wrong hands, and public health officials need more resources to address the problem.

Due to undesirable psychotropic actions, most synthetic cannabinoids have never received approval for therapeutic use in humans. However, these undesirable properties have not deterred the distribution of quasilegal formulations to drug abusers in the form of herbal mixtures commonly branded as 'K2' or 'Spice'. Although many of these products are marked as "not for human consumption" or "incense", it is clear to emergency medicine physicians and medical toxicologists that individuals use these products in place of their illegal counterparts.

Hundreds of so-called 'legal' drug mixtures are being sold worldwide through internet sources and at local convenience stores and head shops. Several potent aminoalkylindole, cyclohexylphenol and other synthetic cannabinoids have been detected in products having wide-ranging concentrations and compositions ${ }^{1,2}$. The fact that these new designer drugs often skirt existing regulation and avoid detection in standardized assays is particularly attractive to those seeking a 'legal high'.

Data recently provided by the American Association of Poison Control Centers or published in clinical reports clearly show that K2 and Spice products are increasingly being used and that these agents are causing an alarming rate of injury and death ${ }^{3}$. From January through August this year, US Poison Control Centers consulted on 4,421 synthetic marijuana exposures, representing an increase over the previous year's total by $52 \%$. Relative potency differences along with a total lack of quality control in $\mathrm{K} 2$ products are likely reasons why $\mathrm{K} 2$ use often leads to considerable adverse reactions, including severe anxiety, acute psychotic episodes, visual and auditory hallucinations and seizures. John W. Huffman, the Clemson University chemist who developed a series of synthetic cannabinoids found in $\mathrm{K} 2$ products, recently said, "Using these things is like playing Russian roulette because, we don't have toxicity data, we don't know the metabolites and we don't know the pharmacokinetics"4.

Assessing the impact of new, emerging designer drugs such as synthetic cannabinoids presents a unique challenge for the biomedical research community. Scientists and public health officials must keep up with what seems to be an almost endless supply of structurally distinct compounds of potential abuse. At the same time, they are being asked to characterize both the pharmacology of these synthetics and the extent to which public health is threatened. This challenge is increasingly difficult in light of reductions in governmental discretionary spending. Moreover, enforcement agencies do not have adequate capacity to take on the additional burden of clinical research and clinical sample analysis.

Although not well known throughout biomedical research forums, the Laboratory Response Network (LRN) offers a potential solution to this problem. The LRN was established by the US Department of Health and Human Services and US Centers for Disease Control and Prevention in accordance with Presidential Decision Directive 39. The mission of the LRN is to develop and support a network of laboratories "that can respond quickly to needs for rapid testing, timely notification, and secure messaging of results associated with acts of biological or chemical terrorism and other high priority public health emergencies." To achieve this objective, LRN laboratories are staffed, trained and equipped to perform complex clinical chemical analyses required to assess human exposure to dangerous chemicals.

One typically thinks of international bioterrorism and chemical warfare when considering the capacities of the LRN network. But when Arkansas legislators began debating whether or not to regulate synthetic cannabinoids, the state health officer took the innovative step of turning to the LRN-Chemical laboratories in Arkansas, because the circumstances of K2 meshed closely with the intended purpose of this network. Resources from private companies, academic institutions, poison control centers, and state and federal agencies were coordinated through the LRN-Chemistry Laboratory to develop a statewide forensic surveillance system, human testing capabilities and a basic research strategy. In part human testing at the LRN facility provided a much enhanced understanding of the pharmacology, metabolism and especially clinical consequences of these products. Ultimately, the collaborative efforts in Arkansas established both the scientific rationale and political support for legislative action. It also contributed to the creation of standards that laboratories worldwide are now using to test human specimens for several cannabinoids found in $\mathrm{K} 2$ products.

Use of LRN-Chemistry laboratories in cooperation with legal drug manufacturers, basic research scientists, clinicians and other stakeholders on behalf of regulators offers a viable solution for developing a national response system capable keeping pace with 'street chemists' who are highly motivated to stay one step ahead of regulatory efforts and laboratory detection capabilities.

Individual states need adequate surveillance systems to track and monitor the distribution of newly emerging drugs of abuse to identify these agents rapidly and facilitate timely research and rational regulatory and educational efforts. Unfortunately, the sheer number of potential drugs, along with countless potential new drug formulations, exceeds state forensic laboratory capacity. Without added capacity and resources, states cannot inform authorities working to curb use and distribution in a timely manner. LRN-Chemistry member laboratories are found in every state and specialize in advanced analytical testing as well as community outreach and education. Given the urgency of the problem of substance abuse, this may be the appropriate time to nationally activate the LRN to provide individual states with the capacity to address the growing public health threat of so-called legal highs.

Jeffery H. Moran is branch chief of the Public Health Laboratory at the Arkansas Department of Health and a research instructor at the University of Arkansas for Medical Sciences.

\footnotetext{
1. Dresen, S. et al. J. Mass. Spectrom. 45, 1186-1194 (2010).

2. Hudson, S. et al. J. Anal. Toxicol. 34, 252-260 (2010).

3. Fattore, L. \& Fratta, W. Front. Behav. Neurosci. 5, 60 (2011).

4. Wang, L. Chem. Eng. News 88, 43 (2010).
} 